\title{
Clar Structure and Fries Set of Fullerenes and (4,6)-Fullerenes on Surfaces
}

\author{
Yang Gao and Heping Zhang \\ School of Mathematics and Statistics, Lanzhou University, Lanzhou, Gansu 730000, China \\ Correspondence should be addressed to Heping Zhang; zhanghp@lzu.edu.cn
}

Received 23 April 2014; Accepted 16 July 2014; Published 5 August 2014

Academic Editor: Qiankun Song

Copyright (C) 2014 Y. Gao and H. Zhang. This is an open access article distributed under the Creative Commons Attribution License, which permits unrestricted use, distribution, and reproduction in any medium, provided the original work is properly cited.

Fowler and Pisanski showed that the Fries number for a fullerene on surface $\Sigma$ is bounded above by $|V| / 3$, and fullerenes which attain this bound are exactly the class of leapfrog fullerenes on surface $\Sigma$. We showed that the Clar number of a fullerene on surface $\Sigma$ is bounded above by $(|V| / 6)-\chi(\Sigma)$, where $\chi(\Sigma)$ stands for the Euler characteristic of $\Sigma$. By establishing a relation between the extremal fullerenes and the extremal $(4,6)$-fullerenes on the sphere, Hartung characterized the fullerenes on the sphere $S_{0}$ for which Clar numbers attain $(|V| / 6)-\chi\left(S_{0}\right)$. We prove that, for a $(4,6)$-fullerene on surface $\Sigma$, its Clar number is bounded above by $(|V| / 6)+\chi(\Sigma)$ and its Fries number is bounded above by $(|V| / 3)+\chi(\Sigma)$, and we characterize the $(4,6)$-fullerenes on surface $\Sigma$ attaining these two bounds in terms of perfect Clar structure. Moreover, we characterize the fullerenes on the projective plane $N_{1}$ for which Clar numbers attain $(|V| / 6)-\chi\left(N_{1}\right)$ in Hartung's method.

\section{Introduction}

Several possibilities of generalizations of the fullerene cages have become a research interest soon after establishing fullerene research itself. Fullerenes are closed carbon-cage molecules made up solely of pentagons and hexagons. One generalization is to consider such trivalent structures with faces of other sizes, that is, fulleroid [1,2], boron-nitrogen fullerene [3-5], (3,6)-fullerene [6, 7], and so forth. Amongst these, boron-nitrogen fullerenes are candidates for fully alternating "inorganic fullerene" cages [3]. By using systematic density-functional tight-binding calculations, Fowler et al. indicated that these hypothetical "inorganic fullerenes" obey an isolated-square rule for boron-nitrogen fullerenes with small number of atoms [3] which is a counterpart of the isolated-pentagon rule [8] for fullerenes. Another generalization is to consider such trivalent structures on other surfaces [9]. Using Euler's formula, we can obtain that the number $f_{5}$ (resp., $f_{4}$ ) of pentagons (resp., quadrilaterals) of a fullerene (resp., (4,6)-fullerene) $F$ on surface $\Sigma$ is equal to $6 \chi(\Sigma)$ (resp., $3 \chi(\Sigma)$, resp.), where $\chi(\Sigma)$ stands for the Euler characteristic of $\Sigma$. It requires that $\chi(\Sigma) \geq 0$. So there are only four possible surfaces on which fullerenes $((4,6)$-fullerenes) can be embedded, namely, the sphere, the projective plane, the torus, and the Klein bottle. The fullerenes $((4,6)$-fullerenes) on these surfaces are called spherical, projective, toroidal, and Klein-bottle fullerenes $((4,6)$-fullerenes), respectively. On other generalizations of fullerenes, we refer to $[2,10,11]$.

The concepts of the Fries number and the Clar number were named after Fries [12] and Clar [13], respectively. An embedding of a graph in a surface is called cellular if each face is homeomorphic to an open disk. A map on a surface $\Sigma$ is a graph embedded cellularly on $\Sigma$. Let $G$ be a 2 connected map. A perfect matching (or Kekulé structure) $M$ of $G$ is a set of edges such that each vertex is incident with exactly one edge in $M$. The faces that have exactly half of their bounding edges in a perfect matching $M$ of $G$ are called alternating faces with respect to $M$. The Fries number Fries $(G)$ of $G$ is the maximum number of alternating faces over all perfect matchings; the Clar number $c(G)$ of $G$ is the maximum number of independent alternating faces over all perfect matchings. Note that the original definitions of the Fries number and the Clar number are the maximum number of alternating hexagonal faces and the maximum number of independent alternating hexagonal faces over all perfect matchings, respectively. The original definitions and 
the definitions we used in this paper coincide when the 2connected map has no other even faces except hexagonal faces. A Fries set is a set of alternating faces with the maximum number of alternating faces. A Clar set is a set of independent alternating faces with the maximum number of alternating faces. If $\mathscr{H}$ is a Clar set of $G$ and $M_{0}$ is a perfect matching of $G-\mathscr{H}$, then we say that $\left(\mathscr{H}, M_{0}\right)$ is a Clar structure of $G$. For a face $f$ of $G$, we say that an edge $e$ in $M$ exits $f$ if $e$ shares exactly one vertex with $f$ and that $e$ lies on $f$ if both vertices of $e$ are incident with $f$.

Both Clar number and Fries number have their chemical significance. The Fries number was proposed as a stability index for hydrocarbon in the early decades of the nineteenth century, whereas the Clar number was later considered as another stability index for hydrocarbon in the 1960s. For buckminsterfullerene $\mathrm{C}_{60}$, Austin et al. think that the source of the stability of it is attributed to the fact that uniquely amongst the 1812 isomers it has a Fries Kekule structure where all hexagons are alternating and all pentagons do not contain any double bond [14]. Ye and Zhang [15] showed that the maximum Clar numbers of spherical fullerenes are $(|V| / 6)-2$ and further characterized all 18 extremal spherical fullerenes among all fullerene isomers of $\mathrm{C}_{60}$. Later, Zhang et al. [16] proposed a combination of Clar number and Kekulé count of spherical fullerenes, as a stability predictor of spherical fullerenes, which turns out to distinguish uniquely the icosahedral $\mathrm{C}_{60}$ from its all 1812 fullerene isomers.

Leapfrog transformation $\mathscr{L}(G)$ for a 2-connected map $G$ on surface $\Sigma$ is usually defined in two ways. One way is first taking a dual of $G$, then truncating the resulting graph $[17,18]$. The other equivalent way is first taking triangulation (or omnicapping) on $G$, then taking a dual of the resulting graph $[17,19,20]$. Triangulation of a map is achieved by adding a new vertex in the center of each face followed by connecting it with each boundary vertex of this face $[17,21]$. Dualization of a map on surface is built as follows: locate a point in the center of each face and join two such points if their corresponding faces share a common edge $[17,20]$. Truncation of a map $G$ is obtained by replacing each vertex $v$ of degree $k$ with $k$ new vertices, one for each edge incident to $v$. Pairs of vertices corresponding to the edges of $G$ are adjacent, and $k$ new vertices corresponding to a single vertex of $G$ are joined in a cycle of length $k[17,18,20]$. If $\mathscr{L}(G)$ is the leapfrog graph of $G$, we say that $G$ is a parent of $\mathscr{L}(G)$. A fullerene (resp., $(4,6)$ fullerene) $F^{\prime}$ is called a leapfrog fullerene (resp., leapfrog $(4,6)$ fullerene) if it is a leapfrog graph of some fullerene (resp., $(4,6)$-fullerene) $F$. In this case, we denote the parent fullerene (resp., $(4,6)$-fullerene) by $\mathscr{L}^{-1}\left(F^{\prime}\right)$, that is, $F=\mathscr{L}^{-1}\left(F^{\prime}\right)$.

The chemical significance of leapfrog transformation is that leapfrog cluster with at least one face of size $3 m+$ 1 or $3 m+2$ has a closed-shell electronic structure, with bounding HOMOs and antibonding LUMOs in Hückel theory, regardless of the electronic configuration of the parent fullerene [22]. The other important fact for leapfrog transformation is that leapfrog fullerenes have at least one Kekule structure containing the maximum number $|V| / 3$ of alternating hexagonal faces and thus to be maximally stable in a localised valence bond picture [23]. Furthermore, the leapfrog spherical fullerenes obey the isolated-pentagon rule, while the leapfrog spherical $(4,6)$-fullerenes obey the isolated-square rule.

The paper is organized as follows. In Section 2, we show that the Clar number for a fullerene on surface $\Sigma$ is bounded above by $(|V| / 6)-\chi(\Sigma)$ and give some other useful results. In Section 3, we prove that the Clar number and Fries number of a $(4,6)$-fullerene on surface $\Sigma$ are bounded above by $(|V| / 6)+\chi(\Sigma)$ and $(|V| / 3)+\chi(\Sigma)$, respectively. We characterize the $(4,6)$-fullerenes on surface $\Sigma$ attaining these two bounds in terms of perfect Clar structure. In Section 4, by using the results in Section 3, we obtain further result that for the toroidal and Klein-bottle fullerenes, the upper bound for Clar number and Fries number always can be attained simultaneously. In Section 5, by establishing a relation between the fullerenes on the projective plane whose Clar numbers attain $(|V| / 6)-\chi(\Sigma)$ and the extremal $(4,6)$ fullerenes on the projective plane whose Clar numbers attain $(|V| / 6)+\chi(\Sigma)$, we also characterize the fullerenes on the projective plane whose Clar numbers attain $(|V| / 6)-\chi(\Sigma)$. In Section 6, we study the relation between the projective fullerenes (resp., (4,6)-fullerenes) attaining maximum Fries number and the spherical fullerenes (resp., (4,6)-fullerenes) attaining maximum Fries number and the relation between the projective fullerenes (resp., (4,6)-fullerenes) attaining maximum Clar number and the spherical fullerenes (resp., $(4,6)$-fullerenes) attaining maximum Clar number.

\section{An Upper Bound for the Clar Number and Fries Number of Fullerenes on Surfaces}

The following lemma is an extension of the result on the sphere in [24] to other surfaces. The proof of it is essentially similar to that in [24]. However, to the completeness of this paper, we present it here.

Lemma 1. Let $F$ be a fullerene on surface $\Sigma$, and let $\mathscr{H}$ be a set of alternating faces with respect to a perfect matching $M$. Then there is an even number of edges in $M$ (possibly 0) exiting any hexagon; there is an odd number of edges in $M$ exiting any pentagon.

Proof. Let $f$ be a face in $F$. If $f$ is a face in $\mathscr{H}$, then no edges in $M$ exit $f$. Suppose that $f$ is not in $\mathscr{H}$. Any face of $\mathscr{H}$ adjacent to $f$ is incident with two adjacent vertices on $f$, as is any edge in $M$ that lies on $f$. Thus if $f$ is a hexagon, then there is an even number of vertices (possibly 0 ) remaining to be covered by the edges in $M$ that exit $f$; if $f$ is a pentagon, then there is an odd number of vertices remaining to be covered by the edges in $M$ that exit $f$.

Zhang and Ye [25] proved that $(|V| / 6)-2$ is the upper bound of Clar number of spherical fullerenes. A new proof was given by Hartung [24]. Gao and Zhang [26] generalized this bound to fullerenes on any surface as the following theorem. Here we give a new proof for this theorem using Hartung's method.

Theorem 2 (see [26]). Let $F$ be a fullerene with $n$ vertices on surface $\Sigma$. Then $c(F) \leq\lfloor n / 6\rfloor-\chi(\Sigma)$. 
Proof. Let $(\mathscr{H}, M)$ be a Clar structure of $F$. Since every face in $\mathscr{H}$ contains six vertices, every edge in $M$ contains two vertices, and every vertex of $F$ is incident with exactly one element of the Clar structure, we have the equation

$$
|\mathscr{H}|=\frac{|V|}{6}-\frac{|M|}{3} .
$$

By Lemma 1 at least one edge in $M$ exits each of the pentagons. There are additional edges in $M$ if one of these edges exits a hexagon. By Euler's formula, a fullerene has exactly $6 \chi(\Sigma)$ pentagons, and thus $|M| \geq 3 \chi(\Sigma)$, where equality holds exactly when all edges from $M$ connect pairs of pentagons. So

$$
c(F)=|\mathscr{H}|=\frac{|V|}{6}-\frac{|M|}{3} \leq \frac{|V|}{6}-\frac{3 \chi(\Sigma)}{3}=\frac{|V|}{6}-\chi(\Sigma) .
$$

We say that a fullerene on surface $\Sigma$ is extremal if the Clar number of the fullerene attains the bound $(|V| / 6)-\chi(\Sigma)$. As a direct corollary of the above theorem, the following result, which is also a generalization of the result on the sphere in [24], gives a necessary condition for the extremality of a fullerene.

Corollary 3. Let $F$ be an extremal fullerene on surface $\Sigma$, and let $(\mathscr{H}, M)$ be a Clar set of $F$. Then there exists a pairing of the $6 \chi(\Sigma)$ pentagons such that each pair is connected by a single edge. The edges in $M$ are only the $3 \chi(\Sigma)$ edges between pairs of pentagonal faces.

Fowler [23] proved that the Fries number for a spherical fullerene $F$ is bounded above by $|V| / 3$ and characterized the spherical fullerenes that attain this bound, that is, the leapfrog spherical fullerenes. Then Fowler and Pisanski [27] generalized this result to trivalent polyhedrons on any surface.

Theorem 4 (see [27]). Let $F$ be a fullerene on surface $\Sigma$. Then Fries $(F) \leq\lfloor|V| / 3\rfloor$.

Proof. For any edge in a perfect matching $M$ of a fullerene $F$, it is contained in at most 2 alternating faces. Since each alternating face has 3 edges in the perfect matching $M$ and $M$ has $|V| / 2$ edges of $F$, we have 3 Fries $(F) \leq 2|M|=|V|$. It implies that Fries $(F) \leq\lfloor|V| / 3\rfloor$ since Fries $(F)$ is an integer.

A perfect Clar structure [27] (or face-only vertex covering [24]) of a map $G$ is a set of vertex-disjoint faces that include each vertex of $G$ once and only once. A perfect matching $M$ is a Fries structure [27] if and only if for every edge, $e$, that belongs to $M$ the two adjacent faces are alternating faces with respect to $M$.

Fowler and Pisanski [27] presented a sufficient condition for a map on a given surface to be a leapfrog graph. Later, in [24], Hartung showed that for planar graphs, the condition is also necessary. We are going to indicate that the sufficient condition is necessary for graphs on any surface.

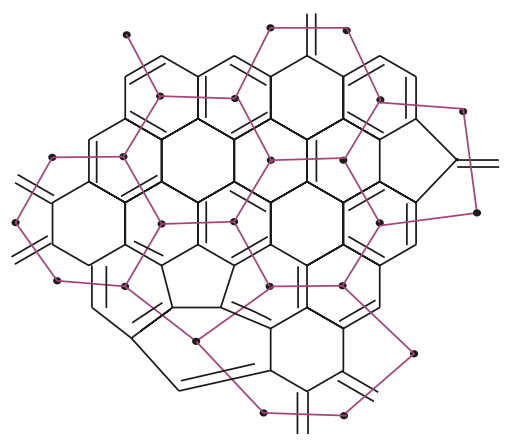

Figure 1: Illustration for the proof of Theorem 5.

Theorem 5. A map $G$ on surface $\Sigma$ is a leapfrog graph if and only if $G$ is 3-regular and has a perfect Clar structure.

Proof. If a map $G$ on surface $\Sigma$ is a leapfrog graph, then each face of the triangulation of the parent graph is triangular. The fact that $G$ is 3-regular follows from the fact that the dual of a triangulation graph is 3-regular. Furthermore, the faces of $G$ corresponding to the faces of the parent graph form a perfect Clar structure of $G$ (see Figure 1).

Conversely, if $G$ is 3 -regular and has a perfect Clar structure $\mathscr{H}$, then $G-E(\mathscr{H})$ is a perfect matching $M$ of $G$. For each alternating face of $G$ with respect to $M$, we add a new vertex at the center of this alternating face; two vertices are adjacent if the corresponding alternating faces are adjacent. Denote the resulting graph by $G^{\prime}$. It is easy to check that $G$ is a leapfrog graph of $G^{\prime}$ on surface $\Sigma$.

The following theorem is first presented by Fowler for spherical fullerenes [23], then generalized by Fowler and Pisanski [27] to trivalent polyhedron on any surface.

Theorem 6 (see [27]). Let $F$ be a fullerene on surface. Then $F$ attains the maximum Fries number $|V| / 3$ if and only if $F$ is a leapfrog fullerene.

For a fullerene $F$ on surface $\Sigma$, if $F$ attains the maximum Fries number $|V| / 3$, then there exists a perfect matching $M$ such that the number of alternating faces is $|V| / 3$, and further $M$ is a Fries structure of $F$. Since each vertex incidents with two alternating faces and a nonalternating face and the nonalternating face contain no edges of $M$, all nonalternating faces form a perfect Clar structure of $F$.

The following theorem is a natural generalization of a similar result on planar graphs in [24] and the proof is similar too.

Theorem 7. Given a bipartite map G on surface with partition $V=(U, W)$. Then the sets of faces of $\mathscr{L}(G)$ corresponding to $U$, $W$, and faces set of $G$ form a face 3-coloring of $F$.

Proof. Since the set of faces in $G$ is corresponding to a perfect Clar structure $\mathscr{H}$ of $\mathscr{L}(G)$, the remaining faces in $\mathscr{L}(G)$ correspond to vertices in $G$. Denote the faces set of $\mathscr{L}(G)$ corresponding to $U$ and $W$ by $\mathscr{U}$ and $\mathscr{W}$, respectively. Since $G$ 
is bipartite, each face of $\mathscr{L}(G)$ is bounded by faces alternating from $\mathcal{U}$ and $\mathscr{W}$. By Theorem 5, any leapfrog graph is 3regular. Thus each vertex in $\mathscr{L}(G)$ is incident with exactly one face from each of sets $\mathscr{U}, \mathscr{W}$, and $\mathscr{H}$.

It is well known that Eulerian triangulation of the plane is 3-colorable. This implies that, for a 3-regular planar graph $G, G$ is face 3-colorable is equivalent to $G=\mathscr{L}\left(G^{\prime}\right)$ for some planar bipartite graph $G^{\prime}$ [24]. Using the same method in [24], we generalize this result to 3-regular maps on any surface as follows.

Theorem 8. Let $G$ be a 3-regular map on surface $\Sigma$. Then the following three statements are equivalent.

(1) $G$ is face 3-colorable.

(2) $G=\mathscr{L}\left(G^{\prime}\right)$ for some bipartite map $G^{\prime}$ on surface $\Sigma$.

(3) G has three different perfect Clar structures.

Proof. (1) $\Rightarrow$ (2) Since $G$ is a 3-regular map, each vertex is incident with a face of each color and each color class is a perfect Clar structure of $G$. By Theorem 5, there exists a graph $G^{\prime}$ such that $G$ is the leapfrog graph of $G^{\prime}$. The faces of one color class in $G$ are one-to-one correspondence to all faces in $G^{\prime}$. Other faces in $G$ are one-to-one correspondence to all vertices in $G^{\prime}$. $G^{\prime}$ is a bipartite map since two vertices are adjacent in $G^{\prime}$ if and only if corresponding faces are adjacent in $G$.

(2) $\Rightarrow$ (1) If $G$ is the leapfrog graph of a bipartite map $G^{\prime}$ on surface $\Sigma$, then the sets of faces of $G$ corresponding to the bipartite partition of $G^{\prime}$ together with the set of faces of $G$ corresponding to the set of faces of $G^{\prime}$ form a face 3-coloring of $G$.

(1) $\Rightarrow$ (3) Obvious.

$(3) \Rightarrow(1)$ For any face of $G$ there is at most one way to adjoin new faces in order to build a perfect Clar structure. So each face belongs to at most one perfect Clar structure. If $G$ has three different perfect Clar structures, then since $G$ is 3regular, each vertex is incident with a face in each perfect Clar structure and three different perfect Clar structures cover each face of $G$ exactly once. So these three different perfect Clar structures of $G$ form a face 3-coloring of $G$.

\section{An Upper Bound for the Clar Number and Fries Number of $(4,6)$-Fullerenes on Surfaces}

The following theorem, presenting an upper bound of Clar number for $(4,6)$-fullerenes on surface $\Sigma$, is a counterpart of Theorem 2 for fullerenes on surface $\Sigma$.

Theorem 9. Let $B$ be a $(4,6)$-fullerene on surface $\Sigma$. Then $c(B) \leq\lfloor|V| / 6\rfloor+\chi(\Sigma)$.

Proof. Let $(\mathscr{H}, M)$ be a Clar structure of $B$. Suppose that $\mathscr{H}$ contains $p$ quadrilaterals, since every face in $\mathscr{H}$ other than quadrilateral faces contains six vertices, every edge in $M$ contains two vertices, and every vertex of $B$ is incident with exactly one element of the Clar structure, we have the equation

$$
6(|\mathscr{H}|-p)+4 p+2|M|=|V|
$$

So

$$
|\mathscr{H}|=\frac{|V|}{6}-\frac{|M|}{3}+\frac{p}{3} .
$$

Since $|M|$ is at least 0 and $p$ is at most $3 \chi(\Sigma)$, we have

$$
c(B)=|\mathscr{H}|=\frac{|V|}{6}-\frac{|M|}{3}+\frac{p}{3} \leq \frac{|V|}{6}+\chi(\Sigma) .
$$

Let $B$ be a $(4,6)$-fullerene on surface $\Sigma$. We say that a $(4,6)$ fullerene is extremal if the Clar number of the $(4,6)$-fullerene attains the bound $(|V| / 6)+\chi(\Sigma)$. For a Clar structure $(\mathscr{H}, M)$ of a $(4,6)$-fullerene, if $M=\emptyset$, we denote $(\mathscr{H}, M)$ by $\mathscr{H}$. Now we are going to characterize the extremal $(4,6)$-fullerenes on surface $\Sigma$.

Theorem 10. Let B be a $(4,6)$-fullerene on surface $\Sigma$. Then the following three statements are equivalent.

(1) $B$ is extremal.

(2) B has a perfect Clar structure containing all quadrilateral faces.

(3) $B$ is a leapfrog $(4,6)$-fullerene.

Proof. (1) $\Rightarrow$ (2) Let $(\mathscr{H}, M)$ be a Clar structure of the extremal (4,6)-fullerene $B$. By Inequality (5), we have $|M|=0$ and $p=3 \chi(\Sigma)$. So $\mathscr{H}$ is a perfect Clar structure containing all $3 \chi(\Sigma)$ quadrilateral faces.

$(2) \Rightarrow(1)$ Let $\mathscr{H}$ be a perfect Clar structure containing all quadrilateral faces in $B$. Then $6(|\mathscr{H}|-3 \chi(\Sigma))+4 \times 3 \chi(\Sigma)=$ $|V|$. So we have $|\mathscr{H}|=(|V| / 6)+\chi(\Sigma)$, and $B$ is extremal.

(2) $\Leftrightarrow(3)$ Obvious.

Theorem 11. Let $B$ be a $(4,6)$-fullerene on surface $\Sigma$. Then Fries $(B) \leq\lfloor|V| / 3\rfloor+\chi(\Sigma)$.

Proof. For any edge in a perfect matching $M$ of a $(4,6)$ fullerene $B$, it is contained in at most 2 alternating faces. Suppose that $\mathscr{I}$ is the set of alternating faces in $B$ with respect to a perfect matching $M$ and $p$ is the number of quadrilaterals in $\mathscr{I}$. Since each alternating hexagon has 3 edges, and each alternating quadrilateral has 2 edges in the perfect matching $M$, we have $3(|\mathscr{I}|-p)+2 p \leq 2|M|=|V|$. It implies that Fries $(B) \leq|\mathscr{I}| \leq\lfloor|V| / 3\rfloor+(p / 3)=\lfloor|V| / 3\rfloor+\chi(\Sigma)$ since Fries $(B)$ is an integer and $p$ is at most $3 \chi(\Sigma)$.

Now we are going to characterize the $(4,6)$-fullerenes on surface $\Sigma$ whose Fries numbers attain the upper bound in Theorem 11 .

Theorem 12. A (4,6)-fullerene $B$ on surface $\Sigma$ attains the maximum Fries number $(|V| / 3)+\chi(\Sigma)$ if and only if $B$ has a perfect Clar structure containing no quadrilaterals. 


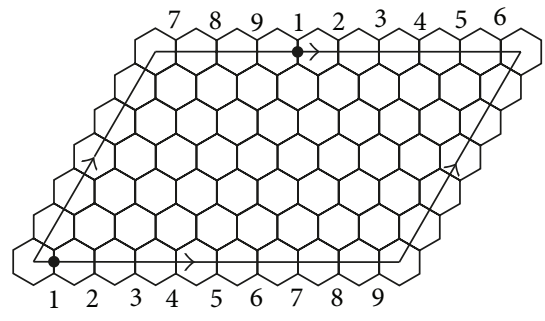

(a)

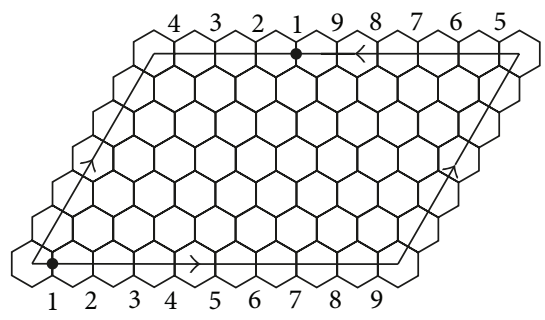

(b)

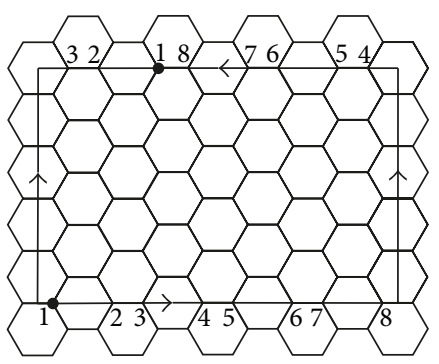

(c)

Figure 2: (a) $H(9,6,3)$; (b) $K(9,6,3)$; (c) $N(8,9,2)$.

Proof. Suppose that $B$ is a $(4,6)$-fullerene on surface $\Sigma$ attaining the maximum Fries number $(|V| / 3)+\chi(\Sigma)$. Then the number of quadrilaterals $p$ in the Fries set $\mathscr{I}$ is equal to $3 \chi(\Sigma)$. Since $B$ has $(|V| / 2)+\chi(\Sigma)$ faces, the remaining $|V| / 6$ faces are all nonalternating hexagonal faces. These $|V| / 6$ nonalternating hexagonal faces form a perfect Clar structure of $B$ contain no quadrilaterals.

Conversely, suppose that $B$ has a perfect Clar structure $\mathscr{H}$ containing no quadrilaterals. Then $|\mathscr{H}|=|V| / 6 . B-E(\mathscr{H})$ is clearly a perfect matching of $B$. Denote this perfect matching by $M$. We prove that each face other than the faces in $\mathscr{H}$ is alternating. To see this, we simply observe that each face $s$ of $B$ different from any hexagon in $\mathscr{H}$ has at least one edge $e$ in $M$, since $M \cup E(\mathscr{H})=E(B)$. Two faces $f_{1}$ and $f_{2}$ connected by $e$ are nonalternating faces with respect to $M$. If $s$ is a quadrilateral face, then the edge $e_{1}$ lying on $s$ that satisfies $d\left(e, e_{1}\right)=1$ also belongs to $M$ since $e$ and $e_{1}$ are exterior of the same nonalternating face, simultaneously. If $s$ is a hexagonal face, then the two edges $e_{1}$ and $e_{2}$ lying on $s$ that satisfy $d\left(e, e_{i}\right)=1(i=1,2)$ also belong to $M$ since $e_{1}$ and $e_{2}$ are exterior edges of two nonalternating faces $f_{1}$ and $f_{2}$, respectively. So $s$ is an alternating face for the perfect matching $M$. Since the number of faces of $B$ other than any hexagon in $\mathscr{H}$ is $(|V| / 3)+\chi(\Sigma), B$ attains the maximum Fries number $(|V| / 3)+\chi(\Sigma)$.

\section{Clar Number and Fries Number of Klein- Bottle Fullerenes and Toroidal Fullerenes}

Since a Klein-bottle fullerene (toroidal fullerene) coincides with a Klein-bottle $(4,6)$-fullerene (toroidal $(4,6)$-fullerene, resp.), we may use the results of the previous section to obtain more information about fullerenes on the Klein bottle and torus whose Clar numbers attain the upper bound in Theorem 9 and fullerenes on the Klein bottle and torus whose Fries numbers attain the upper bound in Theorem 11.

Toroidal fullerenes (resp., Klein-bottle fullerenes) with girth at least 6 are classified into two (resp., five) classes by Thomassen [28], then are reclassified into one new class $H(p, q, t)$ (resp., two new classes $K(p, q, t)$ and $N(p, q, t))$ by Li et al. [29]. For the structures of the $H(p, q, t), K(p, q, t)$, and $N(p, q, t)$, we refer to [26]. Both $H(p, q, t)$ and $K(p, q, t)$ are bipartite graphs with $p q$ faces, $2 p q$ vertices, and $3 p q$ edges, while $N(p, q, t)$ is nonbipartite with $p q / 2$ faces, $p q$

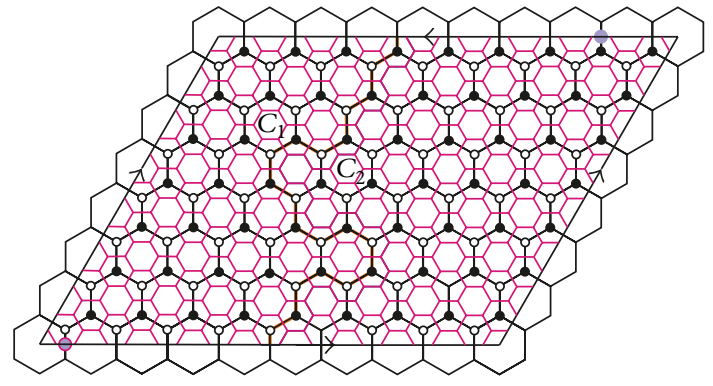

FIGURE 3: Illustration for the first part of proof in Theorem 14.

vertices, and $3 p q / 2$ edges. $H(9,6,3), K(9,6,3)$, and $N(8,9,2)$ are illustrated in Figures 2(a), 2(b), and 2(c), respectively.

Theorem 10, together with Theorem 12, presents some important structure properties of a toroidal or a Klein-bottle fullerene.

Theorem 13. For a toroidal or a Klein-bottle fullerene F, the following four properties are equivalent.

(1) F attains the maximum Fries number $|V| / 3$.

(2) F attains the maximum Clar number $|V| / 6$.

(3) $F$ is a leapfrog fullerene.

(4) F has a perfect Clar structure.

The following theorem reveals an interesting appearance of leapfrog transformation on Klein-bottle fullerenes.

Theorem 14. The leapfrog fullerene of a bipartite Kleinbottle fullerene is nonbipartite, and the leapfrog fullerene of a nonbipartite Klein-bottle fullerene is bipartite.

Proof. We prove the first part of this theorem by presenting an odd cycle in the leapfrog fullerene of a bipartite Klein-bottle fullerene. Given a bipartite Klein-bottle fullerene $K(p, q, t)$, let $C_{1}$ be a noncontractible cycle along the vertical direction of $K(p, q, t)$. Then $C_{1}$ is an even cycle since $K(p, q, t)$ is bipartite. The vertices of $\mathscr{L}(K(p, q, t))$, on either side of $C_{1}$, which are proximal to cycle $C_{1}$ induce a noncontractible cycle $C_{2}$ of $\mathscr{L}(K(p, q, t))$ (see Figure 3). Clearly, the length of $C_{2}$ is equal to the length of $C_{1}$ plus double the times that $C_{1}$ passes 


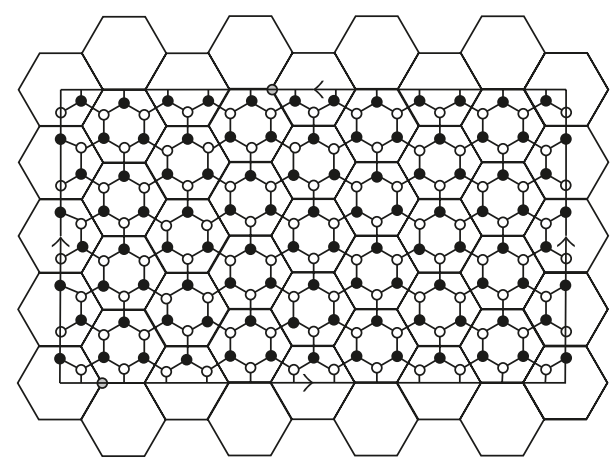

FIGURE 4: Illustration for the second part of proof in Theorem 14.

through the edges of $K(p, q, t)$ and minus one. So $C_{2}$ is an odd cycle in $\mathscr{L}(K(p, q, t))$.

We prove the second part of this theorem by presenting a bipartition in the leapfrog fullerene of a nonbipartite Kleinbottle fullerene $N(p, q, t)$. For each face of the leapfrog fullerene of a nonbipartite Klein-bottle fullerene, which is contained entirely in some face of $N(p, q, t)$, we collect the vertices, along the boundary of this face, into $X$ and $Y$ in the same manner, alternately (see Figure 4). Clearly $X \cup Y$ contains all vertices of the leapfrog graph of $N(p, q, t)$, and both $X$ and $Y$ are independent.

Theorem 14, together with Theorem 8 , implies that none of the bipartite Klein-bottle fullerenes $K(p, q, t)$ are face 3 -colorable and a nonbipartite Klein-bottle fullerene is a leapfrog fullerene if and only if it is face 3-colorable.

The following three theorems in [26] characterize the toroidal and Klein-bottle fullerenes whose Clar numbers attain the maximum Clar number $|V| / 6$.

Theorem 15 (see [26]). $H(p, q, t)$ is extremal if and only if $p \equiv$ $0(\bmod 3)$ and $q \equiv t(\bmod 3)$.

Theorem 16 (see [26]). $K(p, q, t)$ is extremal if and only if $p \equiv$ $0(\bmod 3)$.

Theorem 17 (see [26]). $N(p, q, t)$ is extremal if and only if $q \equiv$ $0(\bmod 3)$.

Combining Theorem 13 and remarks after Theorem 14 with Theorems 15, 16, and 17, respectively, we obtain the following three corollaries.

Corollary 18. For a toroidal fullerene $H(p, q, t)$, the following six properties are equivalent.

(1) $H(p, q, t)$ attains the maximum Fries number $|V| / 3$.

(2) $H(p, q, t)$ attains the maximum Clar number $|V| / 6$.

(3) $H(p, q, t)$ is a leapfrog fullerene.

(4) $H(p, q, t)$ has a perfect Clar structure.

(5) $p \equiv 0(\bmod 3)$ and $q \equiv t(\bmod 3)$.

(6) $H(p, q, t)$ is face 3-colorable.
Corollary 19. For a Klein-bottle fullerene $K(p, q, t)$, the following five properties are equivalent.

(1) $K(p, q, t)$ attains the maximum Fries number $|V| / 3$.

(2) $K(p, q, t)$ attains the maximum Clar number $|V| / 6$.

(3) $K(p, q, t)$ is a leapfrog fullerene.

(4) $K(p, q, t)$ has a perfect Clar structure.

(5) $p \equiv 0(\bmod 3)$.

Corollary 20. For a Klein-bottle fullerene $N(p, q, t)$, the following six properties are equivalent.

(1) $N(p, q, t)$ attains the maximum Fries number $|V| / 3$.

(2) $N(p, q, t)$ attains the maximum Clar number $|V| / 6$.

(3) $N(p, q, t)$ is a leapfrog fullerene.

(4) $N(p, q, t)$ has a perfect Clar structure.

(5) $q \equiv 0(\bmod 3)$.

(6) $N(p, q, t)$ is face 3-colorable.

A map on a surface is called fully-benzenoid if it has a perfect Clar structure consisting only of hexagons. Gutman and Babić [30] gave a characterization of fully-benzenoid hydrocarbons earlier in 1991. Recently Gutman and Salem [31] showed that any fully-benzenoid hydrocarbon has a unique Clar set. Corollary 18, together with Corollaries 19 and 20, implies that fully-benzenoid $K(p, q, t)$ also has this property; however, fully-benzenoid fullerenes $H(p, q, t)$ and $N(p, q, t)$ each have exactly three Clar sets, while by Theorem 2, none of the spherical fullerenes and projective fullerenes is fully-benzenoid.

\section{Extremal Fullerenes on the Sphere and the Projective Plane}

Hitherto, we have already characterized the $(4,6)$-fullerenes on surface $\Sigma$ whose Clar numbers (resp., Fries numbers) attain $(|V| / 6)+\chi(\Sigma)$ (resp., $(|V| / 3)+\chi(\Sigma))$. Fowler and Pisanski [27] characterized the fullerenes whose Fries numbers attain $|V| / 3$. By establishing a relation between the extremal spherical fullerenes and the extremal spherical $(4,6)$-fullerenes on the sphere, Hartung [24] characterized the spherical fullerenes whose Clar numbers attain $(|V| / 6)-\chi(\Sigma)$. So the only question remaining is to characterize the extremal fullerenes on the projective plane. Inspired by Hartung's methods, we characterize the fullerenes on the projective plane whose Clar numbers attain $(|V| / 6)-\chi(\Sigma)$ in this section.

A map on the projective plane is the antipodal quotient of a centrosymmetric spherical map, that is, its vertices, edges, and faces are obtained by identifying antipodal vertices, edges, and faces of the centrosymmetric spherical map. Maps on the projective plane are usually drawn inside a circular frame where antipodal boundary points are identified [9].

For an extremal fullerene $F$ on surface $\Sigma$, let $(\mathscr{H}, M)$ be a Clar structure of $F$. Then the expansion of $F$ is defined as follows: widen each of $3 \chi(\Sigma)$ edges in $M$ between pentagonal 


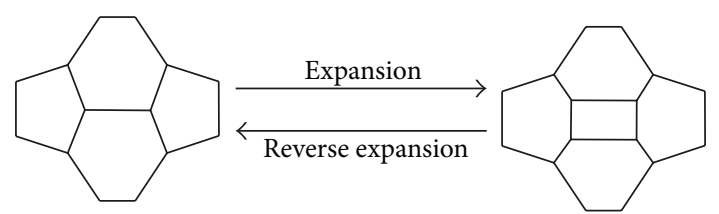

Figure 5: Expansion and reverse expansion.

pairs into a quadrilateral. Under this expansion, we can see that each vertex covered by $M$ becomes an edge, and each pentagon becomes a hexagon (see Figure 5). Denote the resulting $(4,6)$-fullerene by $\mathscr{E}(\mathscr{H}, M)$ and the set of quadrilateral faces by $\mathscr{M}$.

Theorem 21. Let $(\mathscr{H}, M)$ be a Clar structure of an extremal fullerene $F$ on the sphere or the projective plane. Then the expansion $\mathscr{E}(\mathscr{H}, M)$ of $F$ is extremal.

Proof. Since $(\mathscr{H}, M)$ is a Clar structure of an extremal fullerene $F$, by Theorem 2 , we have $|\mathscr{H}|=(|V(F)| / 6)-\chi(\Sigma)$ and $|M|=3 \chi(\Sigma)$. Then $\mathscr{H}^{\prime}=\mathscr{H} \cup \mathscr{M}$ is a set of independent even faces in $\mathscr{E}(\mathscr{H}, M)$ which covers each vertex exactly once. Since $|V(\mathscr{E}(\mathscr{H}, M))|=|V(F)|+6 \chi(\Sigma)$, we have

$$
\begin{aligned}
\left|\mathscr{H}^{\prime}\right| & =|\mathscr{H}|+3 \chi(\Sigma)=\frac{|V(F)|}{6}+2 \chi(\Sigma) \\
& =\frac{|V(\mathscr{E}(\mathscr{H}, M))|}{6}+\chi(\Sigma) .
\end{aligned}
$$

Therefore, $\mathscr{E}(\mathscr{H}, M)$ is extremal.

Corollary 22. Let $F$ be an extremal fullerene on the sphere or the projective plane. Then the expansion $\mathscr{E}(\mathscr{H}, M)$ of $F$ has a perfect Clar structure containing all quadrilateral faces.

Theorem 10 and Corollary 22 imply that expansion of each extremal fullerene on surface $\Sigma$ is always a leapfrog $(4,6)$-fullerene. A natural question is that given a leapfrog $(4,6)$-fullerene $\mathscr{L}(B)$, can we always construct an extremal fullerene $F$ such that $\mathscr{L}(B)$ is the expansion of $F$ ?

For each quadrilateral face of $\mathscr{L}(B)$, we choose a pair of two opposite hexagons adjacent to the quadrilateral, denoted by $\mathscr{P}$. The reverse expansion procedure on the pair $(\mathscr{L}(B), \mathscr{P}), \mathscr{E}^{-1}(\mathscr{L}(B), \mathscr{P})$ is defined as follows: for each quadrilateral face of $\mathscr{L}(B)$, shrink each of the two opposite edges that the quadrilateral shares with paired hexagons into a vertex. We then obtain the graph $\mathscr{E}^{-1}(\mathscr{L}(B), \mathscr{P})$, the reverse expansion of $\mathscr{L}(B)$ with respect to $\mathscr{P}$ (see Figure 5).

The following three theorems are the natural generalizations of the similar results on the sphere in [24]. The proofs are analogous to the corresponding proofs and are omitted here.

Theorem 23. Suppose $\mathscr{L}(B)$ is the leapfrog fullerene of a $(4,6)$ fullerene $B$ on the sphere or the projective plane $\Sigma$. Let $\mathscr{P}$ be the set of hexagons paired on opposite sides of quadrilateral faces of $\mathscr{L}(B)$. The reverse expansion of this pair, $F=\mathscr{E}^{-1}(\mathscr{L}(\mathscr{B}), \mathscr{P})$, is a fullerene if and only if $\mathscr{P}$ is a set of $6 \chi(\Sigma)$ distinct hexagons. When $F$ is a fullerene, $F$ is extremal.
A diagonalization of a $(4,6)$-fullerene $B$ is defined as a choice of diagonal vertices for each quadrilateral face so that no vertex is chosen twice. It follows that $\mathscr{L}(B)$ can be contracted into an extremal fullerene exactly when a diagonalization of $B$ is possible. A perfect diagonalization of a bipartite $(4,6)$-fullerene $B$ is a diagonalization in which all vertices chosen are in the same class of the bipartition.

Theorem 24. Let B be a $(4,6)$-fullerene on the sphere or the projective plane. Then

(1) $B$ has a diagonalization if and only if no vertex meets three quadrilaterals;

(2) $B$ has a perfect diagonalization if and only if $B$ is bipartite and square-isolated. In this case, $B$ has exactly two perfect diagonalizations.

\section{Theorem 25.}

(1) The extremal fullerenes $F$ on the sphere or the projective plane $\Sigma$ with $n$ vertices are in one-to-one correspondence with the diagonalized $(4,6)$-fullerene on $\Sigma$ with $(|n| / 3)+2 \chi(\Sigma)$ vertices.

(2) The extremal fullerenes $F$ on the sphere or the projective plane $\Sigma$ with $n$ vertices that have a Fries set are in oneto-one correspondence with the perfectly diagonalized $(4,6)$-fullerene on $\Sigma$ with $(|n| / 3)+2 \chi(\Sigma)$ vertices.

Perfect diagonalization of a bipartite $(4,6)$-fullerene $B$ on the sphere or the projective plane has been proposed to characterize the extremal fullerenes which has a Fries set of size $|V| / 3$. The hypothesis condition that $B$ is bipartite always holds for $(4,6)$-fullerene on the sphere, since it is well known that a 3-regular planar graph is bipartite if and only if it has only faces of even degree. But it is indispensable for $(4,6)$-fullerenes on the projective plane. In fact, as we will see later, there exist infinitely many nonbipartite $(4,6)$-fullerene on the projective plane. The following theorem is somewhat interesting.

Theorem 26. The leapfrog fullerene of a bipartite $(4,6)$ fullerene on the projective plane is nonbipartite, and the leapfrog fullerene of a nonbipartite $(4,6)$-fullerene on the projective plane is bipartite.

Proof. Suppose B is a bipartite $(4,6)$-fullerene on the projective plane, we prove the first part of this theorem by presenting an odd cycle in $\mathscr{L}(B)$. Let $C$ be any noncontractible cycle of $B$. Then the length of $C$ is even. The vertices of $\mathscr{L}(B)$, on either side of $C$, which are proximal to cycle $C$ induce a cycle $C_{1}$ of $\mathscr{L}(B)$ (see Figure 6(a)). The length of $C_{1}$ is equal to the length of $C$ plus double the times that $P$ passes through edges of $B$ and minus one. So the cycle $C_{1}$ is an odd cycle.

Suppose $B$ is a nonbipartite $(4,6)$-fullerene on the projective plane. We prove the second part of this theorem by proving that there are no odd cycles in $\mathscr{L}(B)$.

For any cycle $C$ in $\mathscr{L}(B)$, if $C$ is a contractible cycle, then $C$ is an even cycle since the vertices of $C$ and the interior of $C$ induce a planar bipartite graph. So let us assume that $C$ is a noncontractible cycle. 


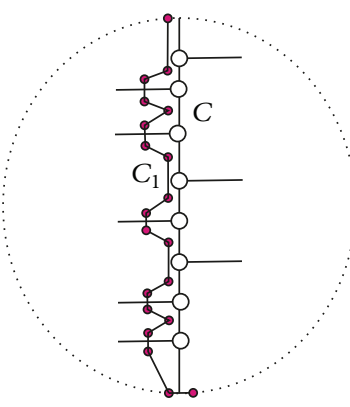

(a)

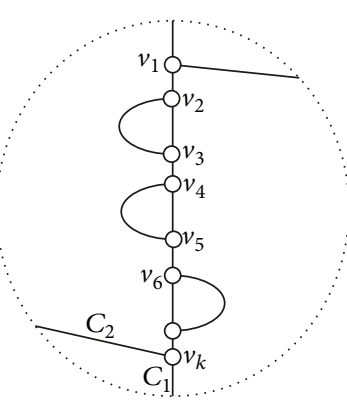

(b)

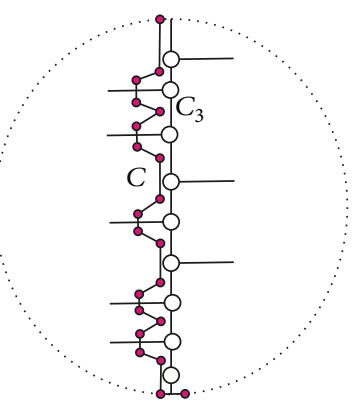

(c)

FIgURE 6: Illustration for the proof of Theorem 26.

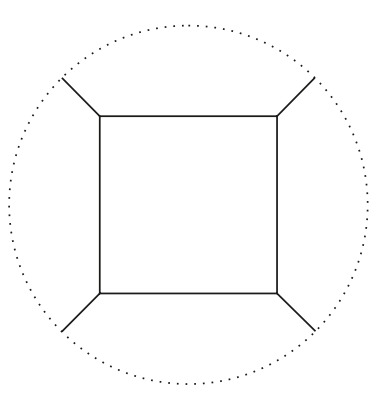

$K_{4}$

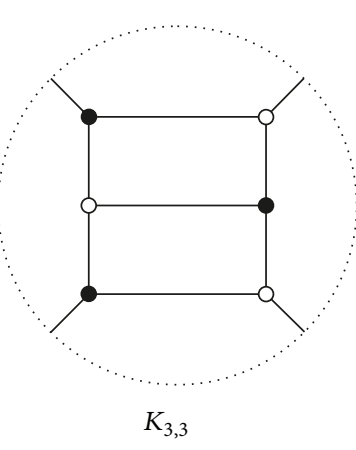

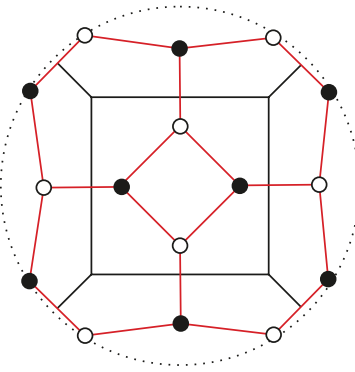

$\mathscr{L}\left(K_{4}\right)$

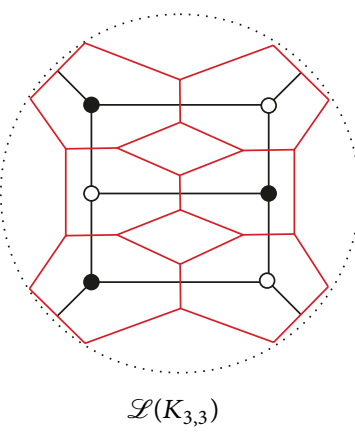

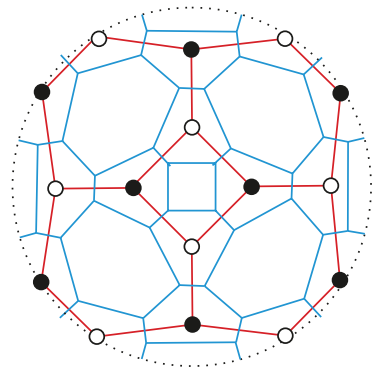

$\mathscr{L}^{2}\left(K_{4}\right)$

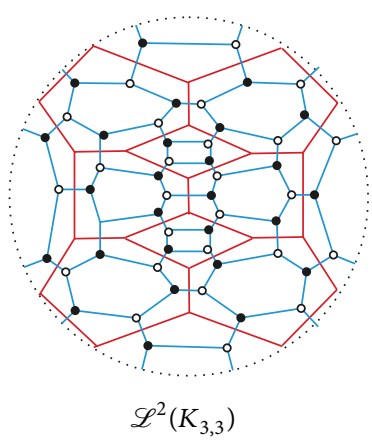

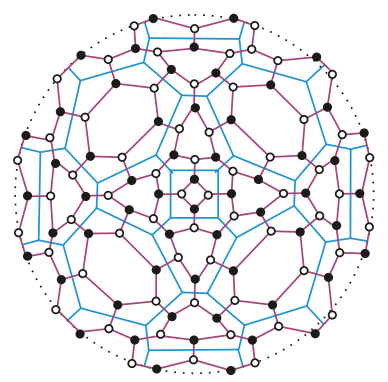

$\mathscr{L}^{3}\left(K_{4}\right)$

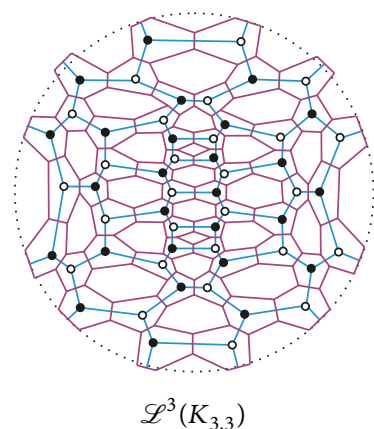

FIGURE 7: The iterated leapfrog fullerenes of $(4,6)$-fullerenes on the projective plane.

Let $C_{1}$ be any noncontractible cycle in $B$. We will show that $C_{1}$ is an odd cycle. Suppose to the contrary that $C_{1}$ is an even cycle. Since $B$ is nonbipartite, there is an odd cycle $C_{2}$ in $B$. It is clear that $C_{2}$ is also a noncontractible cycle of $B$, and the intersection of $C_{1}$ and $C_{2}$ is nonempty. Without loss of generality, we may assume that $v_{1}, v_{2}, \ldots, v_{k}$ are intersection vertices of $C_{1}$ and $C_{2}$ such that only one edge incident with $v_{i}$ on $C_{1}$ does not lie on $C_{2}$ (see Figure 6(b)). Since the lengths of $C_{1}$ and $C_{2}$ have opposite parity, at least one of the boundaries $v_{2} C_{1} v_{3} C_{2} v_{2}, v_{4} C_{1} v_{5} C_{2} v_{4}, \ldots, v_{k} C_{1} v_{1} C_{2} v_{k}$ is of odd length, say $v_{2} C_{1} v_{3} C_{2} v_{2}$. Consider the subgraph $G_{1}$ of $B$ induced by the vertices of $v_{2} C_{1} v_{3} C_{2} v_{2}$ and the interior of the $v_{2} C_{1} v_{3} C_{2} v_{2}$. Clearly, $G_{1}$ is a planar graph and is a subgraph of $B$. Each face of $G_{1}$ except the outer face has length 4 or 6 , thus the length of boundary $v_{2} C_{1} v_{3} C_{2} v_{2}$ is even, a contradiction. So $C_{1}$ is an odd cycle.

The vertices of $B$, on either side of $C$, which are proximal to $C$ induce a noncontractible cycle $C_{3}$ of $B$ (see Figure 6(c)).
The length of $C$ is equal to the length of $C_{3}$ plus double the times that $C$ passes through edges of $B$ minus 1 , thus the lengths of $C$ and $C_{3}$ have the opposite parity. Since $C_{3}$ is an odd cycle, $C$ is an even cycle.

The following theorem presented by Mohar in [32] will be useful for determining the face chromatic number of a $(4,6)$ fullerene on the projective plane.

Theorem 27 (see [32]). Let $G$ be an Eulerian triangulation of the projective plane. Then $\chi(G) \leq 5$ and $G$ has a color factor. Moreover, if $U$ is any color factor of $G$, then

(1) $\chi(G)=3$ if and only if $G-U$ is bipartite;

(2) $\chi(G)=4$ if and only if $G-U$ is not bipartite and does not contain a quadrangulation of the projective plane;

(3) $\chi(G)=5$ if and only if $G-U$ is not bipartite and contains a quadrangulation of the projective plane. Such a quadrangulation is necessarily nonbipartite. 


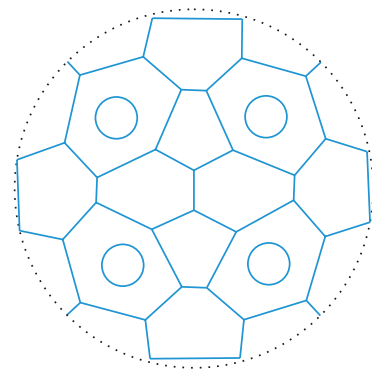

(a)

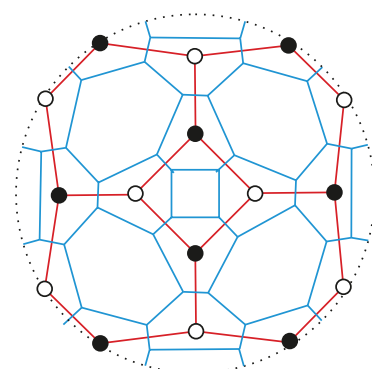

(b)

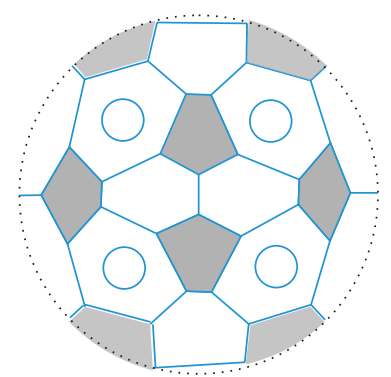

(c)

FIGURE 8: The illustration of constructing extremal projective fullerenes from an extremal projective $(4,6)$-fullerene.

Theorem 27 implies that the unique projective $(4,6)$ fullerene with chromatic number 4 is $K_{4}$. All other projective $(4,6)$-fullerenes are 3 -colorable. This fact further implies that there is a polynomial time algorithm to determine the chromatic number of a projective $(4,6)$-fullerene.

Theorem 28. The face chromatic number of a nonbipartite $(4,6)$-fullerene on the projective plane is 3 , the face chromatic number of a bipartite $(4,6)$-fullerene on the projective plane is 4 except $\mathscr{L}\left(K_{3}\right)$, and the face chromatic number of the bipartite $(4,6)$-fullerene $\mathscr{L}\left(K_{3}\right)$ on the projective plane is 5.

Proof. The theorem holds directly from Theorem 27, the definition of the leapfrog transformation, and the fact that the chromatic number of a map is equal to the face chromatic number of the dual map.

Theorem 23, together with Theorems 25 and 28, generates a method of constructing extremal fullerenes on the sphere and the projective plane from $(4,6)$-fullerene on these surfaces using the leapfrog transformation and the reverse expansion procedure. Given a $(4,6)$-fullerene $B_{0}$ on the sphere or the projective plane, we define iterated leapfrog fullerenes $B_{i}=\mathscr{L}^{i}\left(B_{0}\right)=\mathscr{L}\left(B_{i-1}\right),(i=1,2, \ldots)$. Then all $B_{i}(i \geq 1)$ have a diagonalization, so the reverse expansion $B_{i+1}$ with respect to $3 \chi(\Sigma)$ pairs of hexagons corresponding to a diagonalization of $B_{i}$ generates an extremal fullerene $F_{i}$ on $\Sigma$. By being more specific on the projective plane, if $B_{0}$ is nonbipartite, then each $B_{i}(i \geq 1)$ has a diagonalization and $B_{2 k-1}$ is bipartite and isolated, thus having a perfect diagonalization. If $B_{0}$ is bipartite, then each $B_{i}(i \geq 1)$ has a diagonalization and $B_{2 k}(k \geq 1)$ is bipartite and isolated, thus having a perfect diagonalization (see Figure 7). The reverse expansion with respect to $3 \chi(\Sigma)$ pairs of hexagons corresponding to a perfect diagonalization of $B_{i}$ generates an extremal fullerene $F_{i}$ on $\Sigma$ which also has a Fries set of size $|V| / 3$. All hexagons, which are contained entirely in some hexagon of $B_{i}$, form a Clar set of resulting graph, whereas all faces corresponding to a color class of a bipartite $B_{i}$ form a Fries set of resulting graph. A projective fullerene generated from reverse expansion $\mathscr{L}^{2}\left(K_{4}\right)$ (see Figure $8(\mathrm{~b})$ ) with respect to $3 \chi(\Sigma)$ pairs of hexagons corresponding to a diagonalization (resp., perfect diagonalization) of $\mathscr{L}\left(K_{4}\right)$ is illustrated by Figure 8(a) (resp., Figure 8(c)). Furthermore, two projective fullerenes, which are illustrated in Figures 8(a) and $8(\mathrm{c})$, are the only two projective fullerenes on 30 vertices. It is noticeable that the corresponding centrosymmetric spherical fullerene of the projective fullerene in Figure 8(c) is the famous buckminsterfullerene $\mathrm{C}_{60}$.

\section{Projective Fullerenes versus Spherical Fullerenes}

Since there is a one-to-one correspondence from projective fullerenes to centrosymmetric spherical fullerenes, it is natural to study the relation between the projective fullerenes (resp., (4,6)-fullerenes) attaining maximum Fries number and the spherical fullerenes (resp., (4,6)-fullerenes) attaining maximum Fries number and the relation between extremal projective fullerenes (resp., (4,6)-fullerenes) and extremal spherical fullerenes (resp., (4,6)-fullerenes).

Theorem 29. A map on the projective plane is a leapfrog graph if and only if the corresponding centrosymmetric spherical map is a leapfrog graph.

Proof. Suppose a map $G$ on the projective plane is a leapfrog graph. Then by Theorem 5, $G$ is 3-regular and has a perfect Clar structure $\mathscr{H}$. Since each face of $\mathscr{H}$ is obtained by identifying two antipodal faces of the corresponding centrosymmetric spherical map $G^{\prime}$, all faces in $G^{\prime}$ corresponding to faces of $\mathscr{H}$ in $G$ form a perfect Clar structure $\mathscr{H}^{\prime}$ of $G^{\prime}$. Since each vertex of $G$ is obtained by identifying two antipodal vertices of the corresponding centrosymmetric spherical map $G^{\prime}, G^{\prime}$ is also 3-regular. By Theorem 5, the corresponding centrosymmetric spherical map $G^{\prime}$ of $G$ is a leapfrog graph.

Conversely, suppose the corresponding centrosymmetric spherical map $G^{\prime}$ of $G$ is a leapfrog graph. Then by Theorem 5 , $G^{\prime}$ is 3 -regular and has a perfect Clar structure $\mathscr{H}^{\prime}$. Since the parent graph $\mathscr{L}^{-1}\left(G^{\prime}\right)$ is also a centrosymmetric spherical map and each face of $\mathscr{H}^{\prime}$ is corresponding to a face of $\mathscr{L}^{-1}\left(G^{\prime}\right)$, the antipodal face of each face in $\mathscr{H}^{\prime}$ is also in $\mathscr{H}^{\prime}$. The set of faces obtained by identifying all antipodal faces of $\mathscr{H}^{\prime}$ forms a perfect Clar structure $\mathscr{H}$ of $G$. Clearly, $G$ is 3regular. By Theorem 5, $G$ is a leapfrog graph.

As a corollary of Theorems 10 and 29, we have the following result. 
Corollary 30. A projective $(4,6)$-fullerene is extremal if and only if the corresponding centrosymmetric spherical $(4,6)$ fullerene is extremal.

Combining Corollary 22 with Corollary 30, we have the following result.

Corollary 31. A projective fullerene is extremal if and only if the corresponding centrosymmetric spherical fullerene is extremal.

\section{Conflict of Interests}

The authors declare that there is no conflict of interests regarding the publication of this paper.

\section{Acknowledgment}

This work is supported by NSFC (Grant no. 11371180) and the Fundamental Research Funds for the Central Universities (no. lzujbky-2014-21).

\section{References}

[1] G. Brinkmann and A. W. M. Dress, "Phantasmagorical fulleroids," MATCH: Communications in Mathematical and in Computer Chemistry, vol. 33, pp. 87-100, 1996.

[2] M. Deza and M. D. Sikirić, Geometry of Chemical Graphs: Polycycles and Two-faced Maps, vol. 119 of Encyclopedia of Mathematics and Its Applications, Cambridge University Press, Cambridge, UK, 2008.

[3] P. W. Fowler, T. Heine, D. Mitchell, R. Schmidt, and G. Seifert, "Boron-nitrogen analogues of the fullerenes: the isolatedsquare rule," Journal of the Chemical Society-Faraday Transactions, vol. 92, no. 12, pp. 2197-2201, 1996.

[4] X. Jiang and H. Zhang, "On forcing matching number of boronnitrogen fullerene graphs," Discrete Applied Mathematics, vol. 159, no. 15, pp. 1581-1593, 2011.

[5] R. Yan and F. Zhang, "Clar and sextet polynomials of boronnitrogen fullerenes," MATCH Communications in Mathematical and in Computer Chemistry, vol. 57, no. 3, pp. 643-652, 2007.

[6] P. W. Fowler, P. E. John, and H. Sachs, “( 3,6$)$-cages, hexagonal torodial cages, and their spectra," in Discrete Mathematical Chemistry, P. Hansen, P. Fowler, and M. Zheng, Eds., vol. 51 of DIMACS Series, pp. 139-174, American Mathematical Society, Providence, RI, USA, 2000.

[7] R. Yang and H. Zhang, "Hexagonal resonance of $(3,6)$ fullerenes," Journal of Mathematical Chemistry, vol. 50, no. 1, pp. 261-273, 2012.

[8] P. W. Fowler and D. E. Manolopoulos, An Atlas of Fullerenes, Oxford University Press, Oxford, UK, 1995.

[9] M. Deza, P. W. Fowler, A. Rassat, and K. M. Rogers, "Fullerenes as tilings of surfaces," Journal of Chemical Information and Computer Sciences, vol. 40, no. 3, pp. 550-558, 2000.

[10] M. Deza, M. Dyutur Sikirich, and M. I. Shtogrin, "Fullerenes and disk fullerenes," Russian Mathematical Surveys, vol. 68, no. 4, pp. 69-128, 2013.

[11] M. Deza, M. D. Sikiric, and M. I. Shtogrin, "Fullerene-like spheres with faces of negative curvature," in Diamond and Related Nanostructures, M. V. Diudea and C. L. Nagy, Eds., vol. 6, pp. 251-274, Springer, Dodrecht, Germany, 2013.
[12] K. Fries, "Über byclische verbindungen und ihren vergleich mit dem naphtalin," Annals of Chemistry, vol. 454, pp. 121-324, 1927.

[13] E. Clar, The Aromatic Sextet, Wiley, London, UK, 1972.

[14] S. J. Austin, P. W. Fowler, P. Hansen, D. E. Monolopoulos, and M. Zheng, "Fullerene isomers of $\mathrm{C}_{60}$. Kekulé counts versus stability," Chemical Physics Letters, vol. 228, no. 4-5, pp. 478484, 1994.

[15] D. Ye and H. Zhang, "Extremal fullerene graphs with the maximum Clar number," Discrete Applied Mathematics, vol. 157, no. 14, pp. 3152-3173, 2009.

[16] H. Zhang, D. Ye, and Y. Liu, "A combination of Clar number and Kekulé count as an indicator of relative stability of fullerene isomers of $\mathrm{C}_{60}$," Journal of Mathematical Chemistry, vol. 48, no. 3, pp. 733-740, 2010.

[17] M. V. Diudea, P. E. John, A. Graovac, M. Primorac, and T. Pisanski, "Leapfrog and Related Operations on Toroidal Fullerenes," Croatica Chemica Acta, vol. 76, no. 2, pp. 153-159, 2003.

[18] C. Godsil and G. Royle, Algebraic Graph Theory, Springer, New York, NY, USA, 2001.

[19] G. Brinkmann, P. W. Fowler, and M. Yoshida, "New nonspiral fullerenes from old: generalised truncations of isolatedpentagon-triple carbon cages," Match, vol. 38, pp. 7-17, 1998.

[20] T. Pisanski and M. Randic, "Bridges between geometry and graph theory," in Geometry at Work: Papers in Applied Geometry, vol. 50, pp. 174-194, Mathematical Association of America, Washington, DC, USA, 2000.

[21] R. B. King, Applications of Graph Theory and Topology in Inorganic Cluster and Coordination Chemistry, CRC Press, Boca Raton, Fla, USA, 1993.

[22] D. E. Manolopoulos, D. R. Woodall, and P. W. Fowler, "Electronic stability of fullerenes: eigenvalue theorems for leapfrog carbon clusters," Journal of the Chemical Society, Faraday Transactions, vol. 88, no. 17, pp. 2427-2435, 1992.

[23] P. W. Fowler, "Localised models and leapfrog structures of fullerenes," Journal of the Chemical Society, Perkin Transactions 2, no. 2, pp. 145-146, 1992.

[24] E. Hartung, "Fullerenes with complete Clar structure," Discrete Applied Mathematics, vol. 161, no. 18, pp. 2952-2957, 2013.

[25] H. Zhang and D. Ye, "An upper bound for the Clar number of fullerene graphs," Journal of Mathematical Chemistry, vol. 41, no. 2, pp. 123-133, 2007.

[26] Y. Gao and H. Zhang, "The Clar number of fullerenes on surfaces," MATCH Communications in Mathematical and in Computer Chemistry, vol. 72, pp. 411-426, 2014.

[27] P. W. Fowler and T. Pisanski, "Leapfrog transformations and polyhedra of Clar type," Journal of the Chemical Society, Faraday Transactions, vol. 90, no. 19, pp. 2865-2871, 1994.

[28] C. Thomassen, "Tilings of the torus and the Klein bottle and vertex-transitive graphs on a fixed surface," Transactions of the American Mathematical Society, vol. 323, no. 2, pp. 605-635, 1991.

[29] Q. Li, S. Liu, and H. Zhang, "2-extendability and $k$-resonance of non-bipartite Klein-bottle polyhexes," Discrete Applied Mathematics, vol. 159, no. 8, pp. 800-811, 2011.

[30] I. Gutman and D. Babić, "Characterization of all-benzenoid hydrocarbons," Journal of Molecular Structure: THEOCHEM, vol. 251, pp. 367-373, 1991. 
[31] I. Gutman and K. Salem, "A fully benzenoid system has a unique maximum cardinality resonant set," Acta Applicandae Mathematicae, vol. 112, no. 1, pp. 15-19, 2010.

[32] B. Mohar, "Coloring Eulerian triangulations of the projective plane," Discrete Mathematics, vol. 244, no. 1-3, pp. 339-343, 2002. 


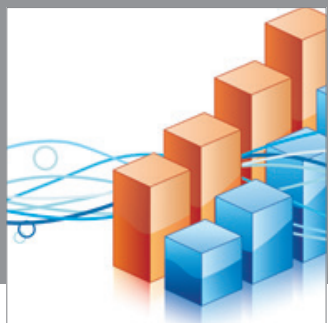

Advances in

Operations Research

mansans

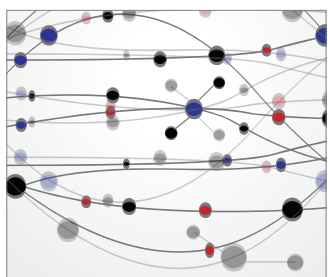

The Scientific World Journal
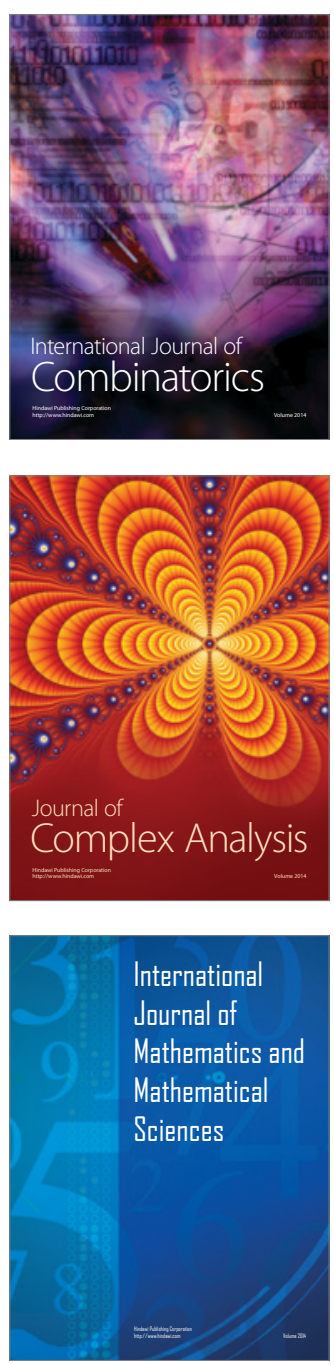
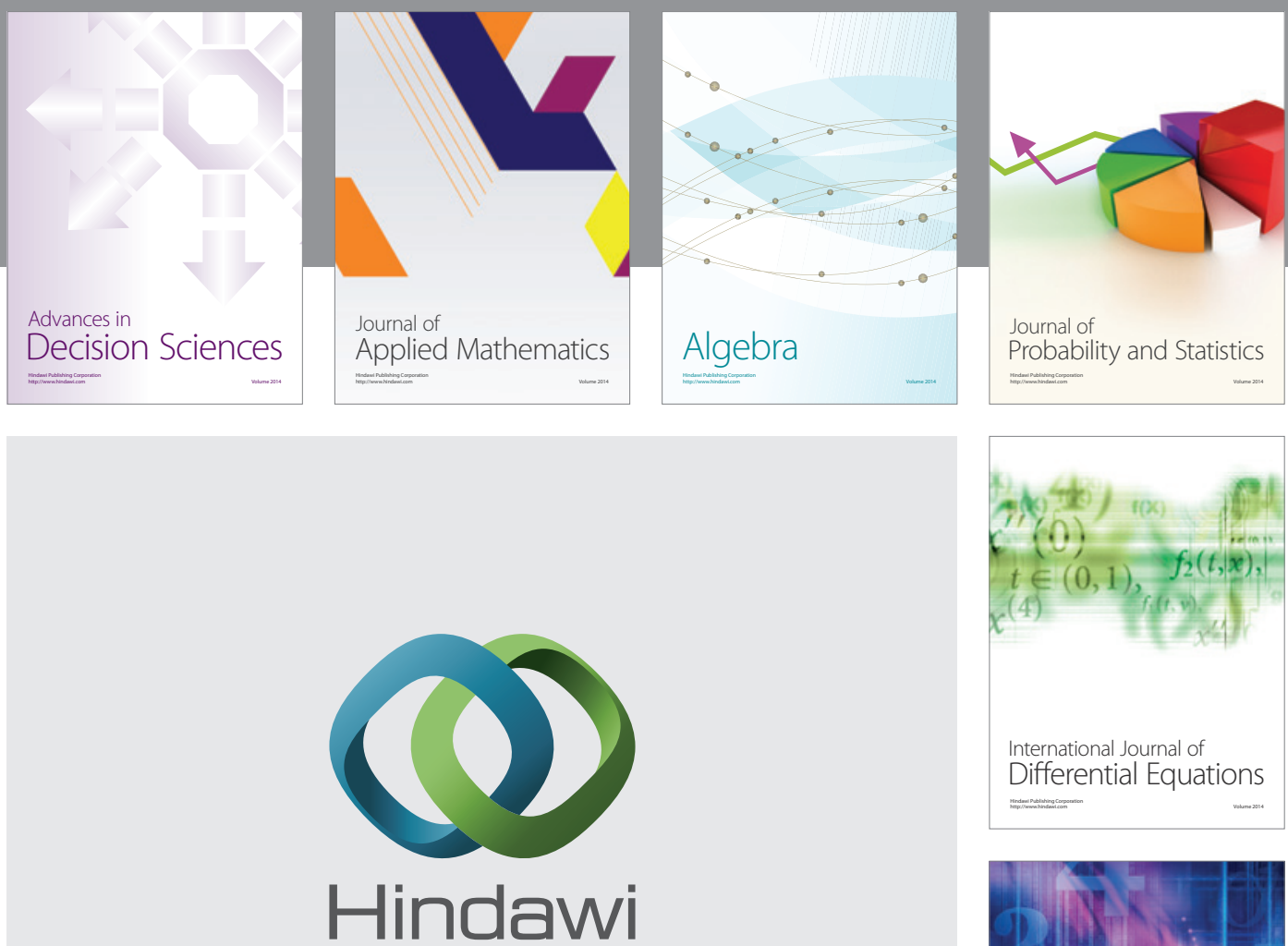

Submit your manuscripts at http://www.hindawi.com
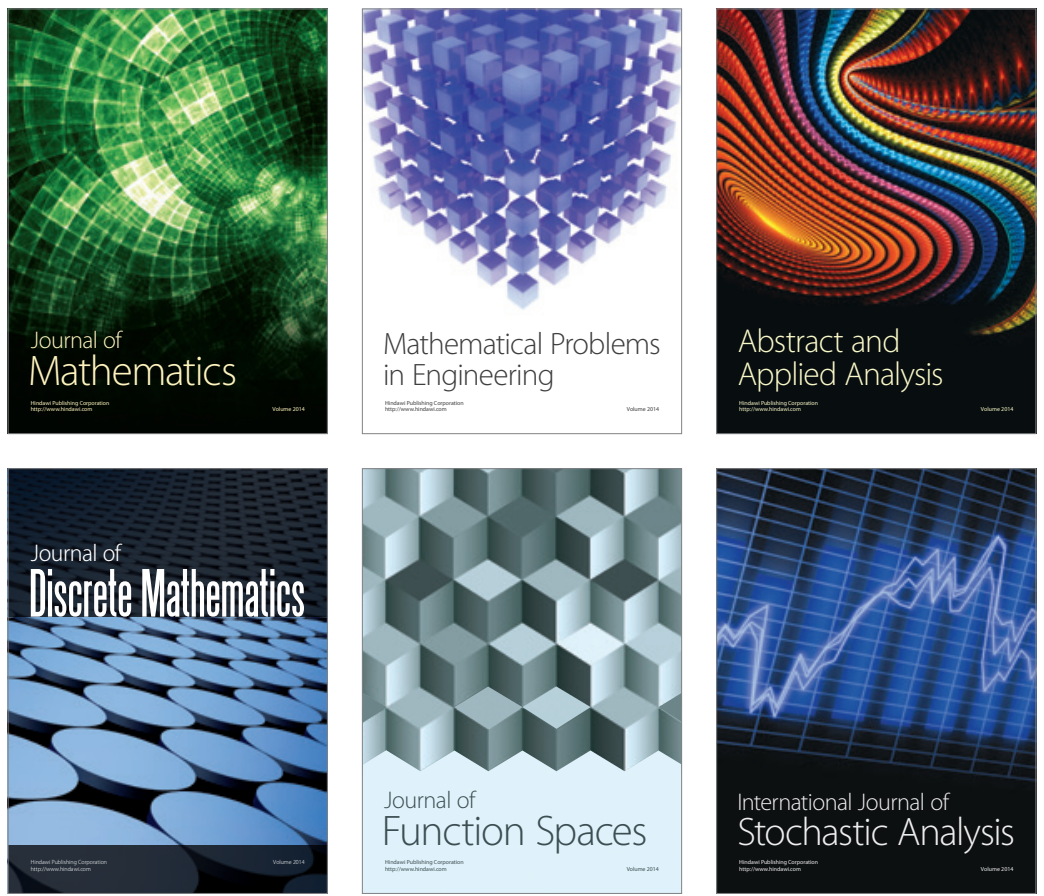

Journal of

Function Spaces

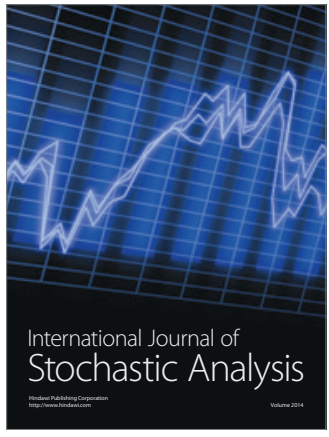

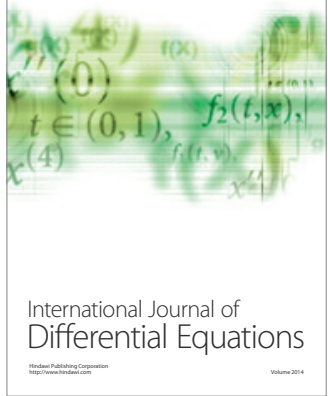
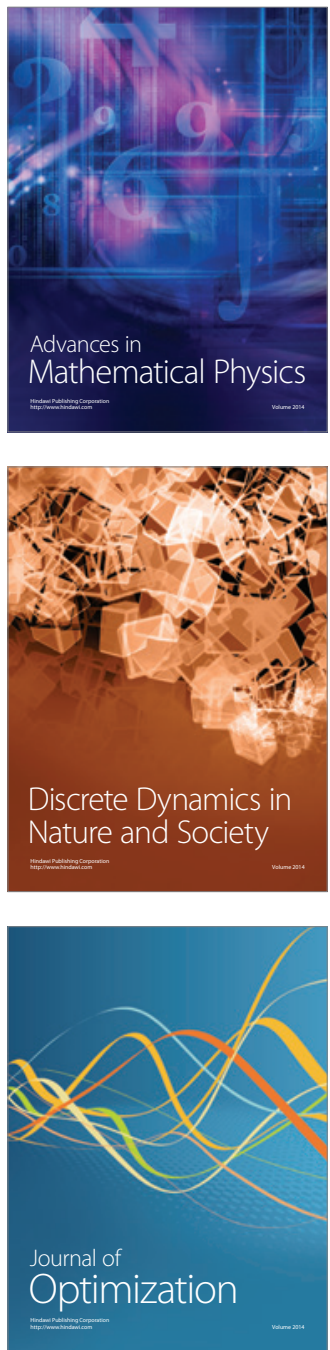